\title{
Emotional memories are not all created equal: Evidence for selective memory enhancement
}

\author{
Adam K. Anderson, ${ }^{1,2,4}$ Yuki Yamaguchi, ${ }^{3}$ Wojtek Grabski, ${ }^{1}$ and Dominika Lacka ${ }^{1}$ \\ ${ }^{1}$ Department of Psychology, University of Toronto, Toronto, Ontario, M5S 3 G 3 Canada; ${ }^{2}$ Rotman Research Institute, Baycrest \\ Centre for Geriatric Care, Toronto, Ontario, M6A $2 E 1$ Canada; ${ }^{3}$ Department of Psychology, Stanford University, \\ Stanford, California 94305, USA
}

\begin{abstract}
Human brain imaging studies have shown that greater amygdala activation to emotional relative to neutral events leads to enhanced episodic memory. Other studies have shown that fearful faces also elicit greater amygdala activation relative to neutral faces. To the extent that amygdala recruitment is sufficient to enhance recollection, these separate lines of evidence predict that recognition memory should be greater for fearful relative to neutral faces. Experiment 1 demonstrated enhanced memory for emotionally negative relative to neutral scenes; however, fearful faces were not subject to enhanced recognition across a variety of delays (15 min to 2 wk). Experiment 2 demonstrated that enhanced delayed recognition for emotional scenes was associated with increased sympathetic autonomic arousal, indexed by the galvanic skin response, relative to fearful faces. These results suggest that while amygdala activation may be necessary, it alone is insufficient to enhance episodic memory formation. It is proposed that a sufficient level of systemic arousal is required to alter memory consolidation resulting in enhanced recollection of emotional events.
\end{abstract}

We tend to remember poignant, emotional events far better than mundane, trivial ones. From births and deaths of loved ones, to weddings and graduations, those events with increased emotional significance achieve prominence in our memory (Christianson 1992; Berntsen and Rubin 2004). Numerous studies have revealed a critical role for the human amygdala in the enhanced episodic memory accompanying emotional events (Cahill et al. 1995, 1996; Adolphs et al. 1997, 2005b; Phelps and Anderson 1997; LaBar and Phelps 1998; Hamann et al. 1999; Canli et al. 2000; Kilpatrick and Cahill 2003; Dolcos et al. 2004; Kensinger and Corkin 2004; Sharot et al. 2004). Increased amygdala activation during stimulus encoding specifically correlates with superior memory for individual emotional stimuli (Canli et al. 2000; Dolcos et al. 2004). These findings are consistent with evidence from nonhuman animals that amygdala increases long-term memory formation by modulating long-term potentiation (Ikegaya et al. 1995; Richter-Levin and Akirav 2000; Akirav and Richter-Levin 2002) and thus, the consolidation of memory traces in brain regions involved in different forms of memory (McGaugh et al. 1996; Packard and Cahill 2001). In particular, in humans, amygdala-hippocampal interactions have been demonstrated to support the enhancement of episodic memory for emotionally significant events (Hamann et al. 1999; Kilpatrick and Cahill 2003; Dolcos et al. 2004).

In addition to its critical role in enhancing episodic memory for emotional events, lesion studies have shown patients with amygdala damage have difficulty recognizing fearful facial expressions (Adolphs et al. 1994, 1999, 2005a; Broks et al. 1998; Anderson and Phelps 2000; Anderson et al. 2000). Numerous neuroimaging studies have now shown that the amygdala is activated more when subjects view fearful faces than neutral, happy, angry, or disgusted faces (Breiter et al. 1996; Morris et al. 1996; Phillips et al. 1998; Whalen et al. 1998, 2001; Hariri et al.

\footnotetext{
${ }^{4}$ Corresponding author.

E-mail anderson@psych.utoronto.ca; fax (416) 978-4811.

Article published online before print. Article and publication date are at http:// www.learnmem.org/cgi/doi/10.1101/lm.388906.
}

2000, 2002; Anderson et al. 2003a). The robustness of this amygdala recruitment is demonstrated in how fearful faces have been shown to evoke amygdala activation to a degree even greater than that evoked by complex emotional scenes (Hariri et al. 2002).

The importance of these two separate neural observations is that they converge on a novel behavioral prediction regarding the expression of the emotional enhancement of memory (EEM). Given amygdala activation is a critical predictor of enhanced memory for emotional stimuli, memory for fearful faces should be similarly enhanced relative to neutral events. This evidence would suggest that EEM might be reduced to the simple neurobiological prerequisite of increased amygdala activation. That is, if the amygdala is "turned on" in association with a stimulus, this stimulus will be subject to later enhanced memory. As such, differential amygdala activation for fearful relative to neutral faces should then result in their being more likely remembered than forgotten.

In contrast, if memory were not enhanced for fearful faces, then this would suggest that amygdala activation might be a necessary, but insufficient cause for EEM. This would indicate that in addition to amygdala recruitment, some supplemental cognitive and/or affective factor(s) are required to result in enhanced recollection of events with emotional significance. For instance, one factor of importance may be the influence of arousal around the time of stimulus encoding (Anderson et al. 2006). Previous studies of enhanced emotional memory demonstrate the important role of arousal accompanying emotional stimuli (Phelps et al. 1997; LaBar and Phelps 1998; Buchanan et al. 1999; Buchanan and Lovallo 2001; Hamann et al. 2002). Facial expressions, such as fear, may evoke more limited systemic arousal (Lang et al. 1993). If amygdala activation is sufficient to alter the course of episodic memory, then activation of the amygdala alone, independently of higher levels of subjective or autonomic indices of arousal, should be sufficient for enhanced memory, predicting EEM for fearful relative to neutral faces. In contrast, arousal may be a critical prerequisite for the amygdala to influence memory (Cahill and McGaugh 1998; Ca- 
A

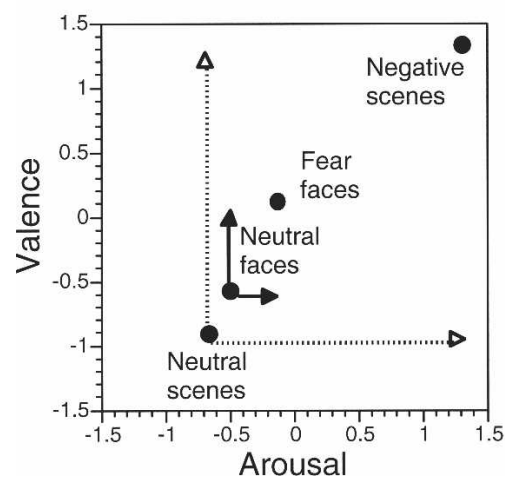

B

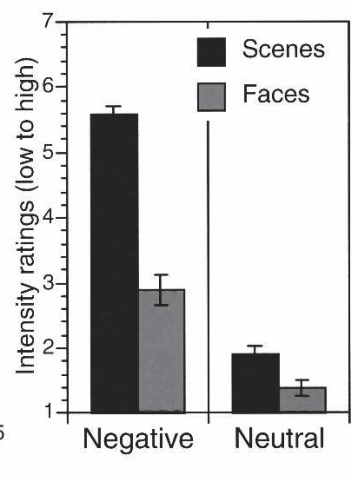

Figure 1. (A) Pre-experimental ratings of valence and intensity (i.e. arousal) of emotional experience during viewing faces and scenes. Valence is along the $y$-axis and arousal the $x$-axis. Values are presented in z-transformed units. Arrows denote the relative distance between fear and neutral faces (solid line) and negative and neutral scenes (dotted line) according to valence and arousal. Negative scenes used in the experiment were rated as both more arousing and negative. (B) Experimental ratings of emotional intensity experienced during encoding of faces and scenes. Negative scenes were reported as resulting in the most intense emotional response. Negative faces $=$ fear expressions.

hill et al. 2003; McGaugh 2004; Okuda et al. 2004), predicting EEM for highly arousing events, but not for fearful faces.

\section{Results}

\section{Experiment 1}

\section{Emotional intensity ratings}

Rating data were submitted to a 3-way ANOVA with delay, stimulus type (faces vs. scenes), and emotion (negative vs. neutral) submitted as separate factors. Scenes were rated as more emotionally intense than faces, $F_{(1,43)}=123.69, P<0.001$. As shown in Figure 1, this main effect was largely due to an interaction between stimulus type and emotion, $F_{(1,43)}=57.33, P<0.001$, with negative scenes rated as more intense than all other stimulus types, $F_{(1,43)}=430.88, P<0.001$, and in particular, greater than fear faces, $F_{(1,43)}=176.71, P<0.001$. Although smaller in magnitude, ratings of fear faces remained greater than neutral faces, $F_{(1,43)}=54.03, P<0.001$, and neutral scenes, $F_{(1,43)}=21.71$, $P<0.001$. There was a trend for arousal ratings across stimulus types to be higher for the shortest delay group, $F_{(1,43)}=3.10$, $P<0.06$; however, the lack of a significant delay $\mathrm{x}$ stimulus type $\mathrm{x}$ emotion interaction, $F_{(1,43)}<1$, suggests that the three delay groups demonstrated similar greater reported intensity of emotional response to negative scenes relative to other stimulus types.

\section{Scene memory}

As illustrated in Figure 2A,B, the emotional content of scenes enhanced remember but not familiar responses. Remember and familiar response data were submitted to two independent ANOVAs with delay and scene type as separate factors. Corrected "remember" responses (remember Hits - remember false alarms $[\mathrm{FA}]$ ) revealed that recollection significantly depended on delay, $F_{(2,43)}=8.75, P<0.001$, with recognition accuracy decreasing linearly with increasing delay, $F_{(1,43)}=17.47, P<0.001$. Overall recollection was more accurate for negative than neutral scenes, $F_{(1,43)}=14.25, P<0.001$. An interaction between scene type with delay, $F_{(1,43)}=9.04, P<0.001$, revealed that delay had a greater than twofold reduction in recollection of neutral, $F_{(1,43)}=27.92$, $P<0.001$, (41\% reduction) compared with negative scenes, $F_{(1,43)}=6.36, P<0.02$ (a $19 \%$ reduction). The recollection advantage for negative relative to neutral scenes was significantly different from neutral only at the longest test delay, $F_{(1,43)}=29.44$, $P<0.001$. In contrast with prior studies, the long delay needed to demonstrate EEM likely reflects the use of a relatively small number of studied items, resulting in relative resistance of forgetting for neutral items.

Corrected "familiar" responses (familiar Hits - familiar FA) depended on delay, $F_{(2,43)}=5.72, P<0.007$, increasing with increasing delay, $F_{(1,43)}=10.05, P<0.003$. As illustrated in Figure $2 \mathrm{~B}$, in contrast with remember responses, familiar responses did not significantly differ between stimulus types across delay, $F_{(2,43)}=2.53, P<0.12$, or in a delay-dependent manner, $F_{(2,43)}<1$, although this may in part reflect a floor level responding.

\section{Face memory}

As illustrated in Figure 3A,B, neither remember or familiar responses indicated enhanced recognition memory for fear faces. Remember and familiar response data were submitted to two independent ANOVAs with delay and face type as separate factors. Corrected "remember" responses significantly depended on delay, $F_{(2,43)}=3.79, P<0.04$, with recognition accuracy decreasing linearly with increasing delay, $F_{(1,43)}=6.65, P<0.02$. Recollection did not significantly differ for fear and neutral faces across delays, $F_{(2,43)}<1$, or in a delay-dependent manner, $F_{(2,43)}<1$. This lack of differentiation was not due to floor-level performance at the longest delay. We next performed a median split analysis on the longest delay group based on neutral face memory performance, submitting individuals with high versus low face memory to an additional ANOVA with face type as a separate factor. This analysis yielded corrected recognition for neutral faces well above floor levels (>56.5\%), yet accuracy for fear faces did not significantly differ from neutral in individuals with either high- or low-memory accuracy, $F_{(1,12)}<1$.

Despite similar magnitudes of remember responses for fearful and neutral faces, the qualitative character of memory may have differed, favoring familiarity. However, familiar responses like remember responses did not significantly differ between face types across delays, $F_{(2,43)}<1$, or in a delay-dependent manner, $F_{(2,43)}<1$.

\section{Learning \& Memory} www.learnmem.org
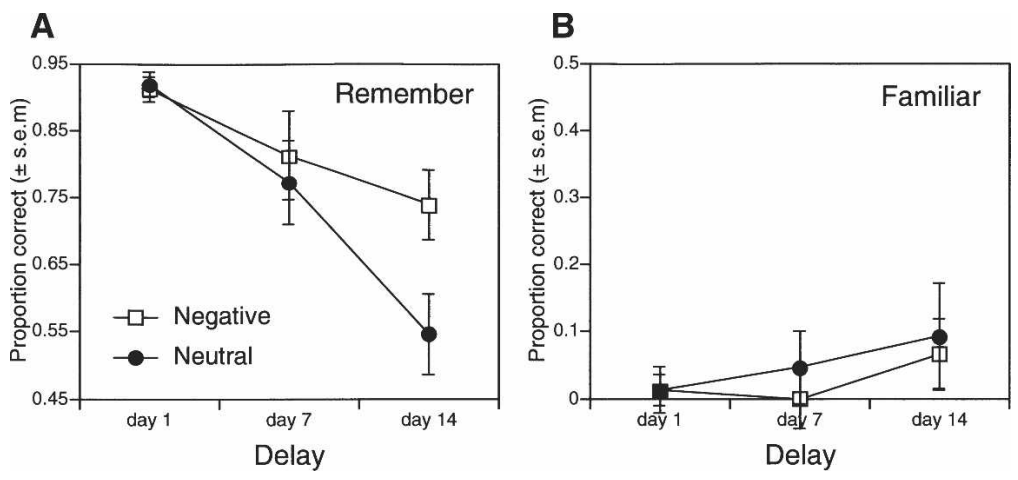

Figure 2. Recognition memory for scenes. Proportion corrected (A) "remember" and (B) "familiar" responses for negative and neutral scenes at different test delays. 
A

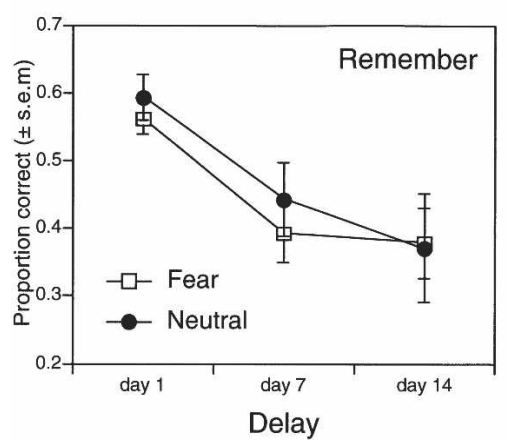

B

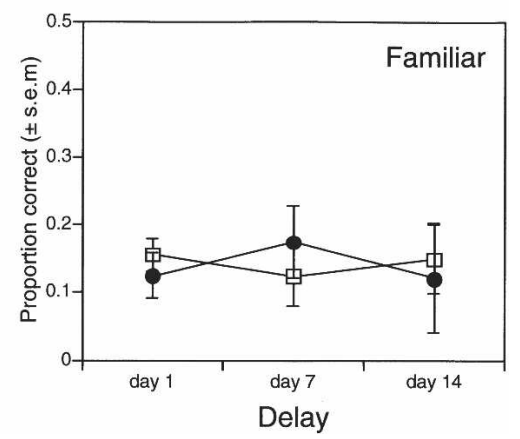

Figure 3. Recognition memory for faces. Proportion corrected $(A)$ "remember" and $(B)$ "familiar" responses for fear and neutral faces at different test delays.

\section{Differential forgetting: Faces and scenes}

Recognition memory for scenes was more accurate than faces even at the shortest delay. Although performance was sufficiently off floor levels to permit differentiation of memory for fear and neutral faces, baseline differences in memory may obscure the magnitude of differential forgetting across stimulus types. To examine this more closely, corrected memory at the longest delay for faces and scenes were proportionally scaled relative to mean corrected memory at the shortest delay for each stimulus type. These scores represented the proportional magnitude of differential forgetting for all stimulus types and were submitted to an additional ANOVA with emotion (negative vs. neutral) and stimulus type (faces vs. scenes) as separate factors. As illustrated in Figure 4, rate of forgetting did not differ between neutral faces and scenes. An interaction between emotion and stimulus type, $F_{(1,13)}=4.87, P<0.05$, revealed that compared with all other stimulus types, negative scenes were least susceptible to forgetting, $F_{(1,13)}=14.28, P<0.003$, and in particular, were maintained in memory greater than fearful faces, $F_{(1,13)}=11.65, P<0.004$. In contrast, fearful faces were equally susceptible to forgetting as neutral scenes and faces, $F_{(1,13)}<1$.

\section{Discussion}

Consistent with prior findings, emotionally aversive scenes resulted in enhanced recognition memory. Examination of forgetting curves revealed differential forgetting of neutral relative to negative scenes, with the latter being relatively maintained in memory over a 2-wk delay. In particular, negative scenes were

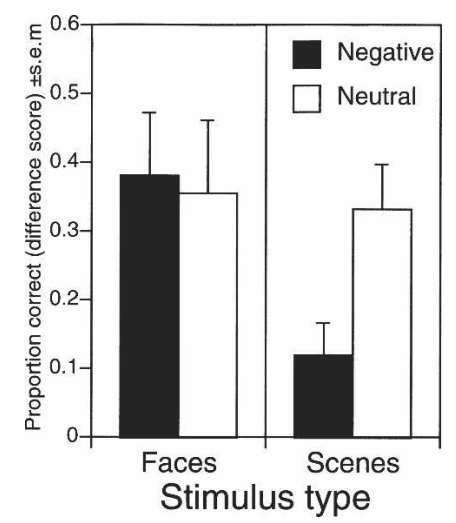

Figure 4. Comparison of degree of forgetting for scenes and faces from day 1 (15 $\mathrm{min}$ ) to $2 \mathrm{wk}$. Proportion corrected recognition difference score ( 2 wk minus day 1 ) for scenes and faces. endowed with an enhanced sense of recollection but not familiarity, consistent with prior studies (Dewhurst and Parry 2000; Ochsner 2000; Kensinger and Corkin 2003). In contrast, we show fearful faces were not subject to enhanced recollection or familiarity across a variety of delays, ranging from $15 \mathrm{~min}$ to $2 \mathrm{wk}$. The failure of EEM for fearful faces was thus unlikely due to not testing at appropriate delays that would reveal memory enhancement. This mnemonic dissociation suggests that greater amygdala response to fear faces may be insufficient to enhance later memory and that additional factors may be necessary for the presence of EEM.

Fear faces and negative scenes differ in many aspects that may be important for the expression of EEM. One potential factor is the presence of systemic physiologic arousal (Cahill et al. 1994). Facial expressions, such as fear, may evoke more limited systemic arousal (Lang et al. 1993), as suggested in the present study by greater self-reported arousal for negative scenes relative to fearful faces. Enhanced emotional memory has been associated with sufficient systemic arousal accompanying emotional stimuli (Phelps et al. 1997; LaBar and Phelps 1998; Buchanan et al. 1999; Buchanan and Lovallo 2001; Hamann et al. 2002; Anderson et al. 2006). Amygdala activation independent of a certain degree of autonomic arousal may be insufficient to alter the course of episodic memory.

\section{Results}

\section{Experiment 2}

Experiment 2 used the galvanic skin response (GSR) to examine whether fearful faces and aversive scenes differed in their capacity to evoke peripheral sympathetic arousal. In addition, the experimental paradigm was altered to reduce the influence of other factors that may have contributed to the failure to find EEM for fearful faces. The emotional intensity ratings task was removed, as the diagnosticity of this task for aversive scenes (i.e., being judged as more intense) may have differentially increased their depth of processing during encoding. Study 1 also used separate encoding sessions for faces and scenes. Studies examining the influence of mixed versus pure study lists have revealed EEM only in the latter case (Dewhurst and Parry 2000). As such, enhanced inter-item associations in pure study lists of faces might reduce the distinctiveness of fear faces, reducing their capacity to result in EEM (Dewhurst and Parry 2000). In study 2, faces and scenes were intermixed during encoding and later test.

\section{Galvanic skin response}

Negative scenes, but not fear faces resulted in a significant increase in autonomic arousal responses relative to neutral events (Figure 5A). GSR data were submitted to a two-way ANOVA, with emotion (negative and neutral) and type (scenes and faces) as separate repeated measures factors. There was a significant main effect of emotion, with negative events associated with greater GSR response relative to neutral, $F_{(1,21)}=7.13, P<0.02$. An interaction between emotion and picture type revealed that the effect of emotion was selective for negative scenes, $F_{(1,21)}=6.03$, $P<0.03$, which resulted in more pronounced responses relative to all other conditions, $F_{(1,21)}=11.30, P<0.003$. Fear faces did not differ from neutral faces, $F_{(1,21)}<1$, or scenes, $F_{(1,21)}=1.33$, $P>0.26$. 
A

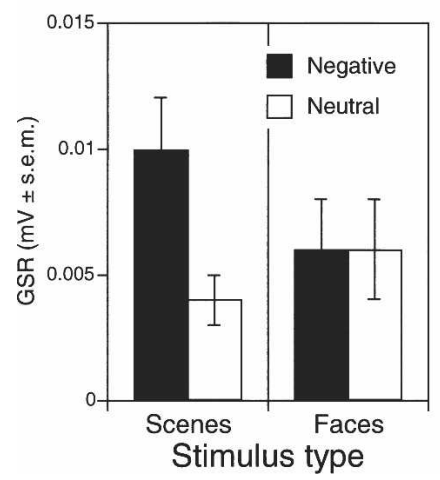

\section{B}

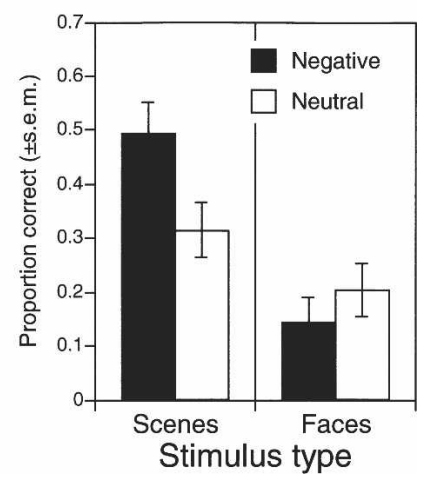

Figure 5. Comparison of sympathetic arousal and recognition memory. ( $A$ ) Galvanic skin responses and $(B)$ Proportion corrected "remember" responses for faces and scenes in Experiment 2.

\section{Recognition memory}

Replicating Experiment 1, negative scenes, but not fear faces resulted in a significant increase in remember responses relative to neutral events after a 2-wk delay (Figure 5B). Corrected remember data for remember and familiar responses were submitted to separate two-way ANOVAs, with emotion (negative and neutral) and type (scenes and faces) as separate repeated measures factors. A main effect of event type, $F_{(1,17)}=34.15, P<0.0001$, revealed that scenes were better remembered than faces. A marginally significant main effect of emotion suggested that negative events were better recollected than neutral, $F_{(1,17)}=4.32, P<0.06$. An interaction between emotion and picture type revealed that the effect of emotion was selective for negative scenes, $F_{(1,17)}=10.18$, $P<0.006$, with negative scenes resulting in more pronounced recollection relative to all other conditions, $F_{(1,17)}=40.19$, $P<0.0001$, including fearful faces, $F_{(1,17)}=44.16, P<0.0001$.

The relatively low levels of recognition memory in particular for faces may have masked differences between neutral and fear faces. To address this, we next performed a median split analysis based on neutral face memory performance, submitting individuals with high versus low neutral face memory to an additional ANOVA with face type as a separate factor. Accuracy for fear relative to neutral faces did not differ depending on whether individuals demonstrated high- or low-memory accuracy, $F_{(1,16)}=1.87, P>0.19$. A similar median split analysis based on fear face memory performance similarly demonstrated that fear and neutral faces' memory did not differ depending on whether individuals demonstrated high- or low-memory accuracy, $F_{(1,16)}<1$.

With respect to potential differences in the qualitative character of memory faces and scenes, an analysis of familiar responses revealed no effects of significance (all $P>0.13$ ). In particular, emotion did not interact with picture type, nor were fear faces judged as more familiar than neutral, $F<1$.

\section{Discussion}

Previous studies have shown that increased amygdala activation during stimulus viewing is strongly associated with enhanced memory for emotional scenes (Cahill et al. 1995, 1996; Hamann et al. 1999; Canli et al. 2000; Kilpatrick and Cahill 2003; Dolcos et al. 2004; Kensinger and Corkin 2004; Sharot et al. 2004). It is also well-established that fearful faces elicit significantly greater amygdala response than neutral faces (Breiter et al. 1996; Morris et al. 1996; Phillips et al. 1998; Whalen et al. 1998, 2001; Hariri et al. 2000, 2002; Anderson et al. 2003a). The present study used these two sources of neuroimaging evidence to make a novel prediction about the behavioral expression of the emotional enhancement of memory (EEM). Specifically, if increased amygdala activation is sufficient to enhance episodic memory, then EEM should be present for fearful faces relative to neutral events. In contrast, Experiment 1 provides evidence of a dissociation in EEM for aversive scenes and fearful faces. Unlike aversive scenes, memory for fearful faces was not better than memory for neutral materials at various delays extending from $15 \mathrm{~min}$ to $2 \mathrm{wk}$ following initial encoding. With respect to the phenomenological quality of memory retrieval, consistent with prior reports (Dewhurst and Parry 2000; Ochsner 2000; Kensinger and Corkin 2003) emotionally aversive scenes specifically enhanced the experience of remembering rather than familiarity (Yonelinas and Levy 2002); in contrast, fearful faces did not enhance either remember or familiar responses. Experiment 2 replicated the above pattern of findings in a mixed-list study design and further demonstrated that aversive scenes but not fearful faces were associated with a significant increase in peripheral sympathetic arousal, as indexed by the galvanic skin response. This parallel dissociation in memory and sympathetic arousal suggests that differential activation of the amygdala may be necessary but insufficient to enhance episodic memory for emotional events.

There are many potential differences between fearful faces and aversive scenes that may contribute to evidence for selective memory enhancement. We begin by considering potential differences in amygdala engagement in processing fearful faces and aversive scenes and then turn to potential differences in associated cognitive and affective processes. First, amygdala activation may have differed in overall magnitude for fear faces and aversive scenes. Although amygdala activation was not measured in this study, this view is not supported by other studies using stimuli similar to those used here that demonstrate even greater amygdala activation to fearful faces than aversive scenes (Hariri et al. 2002). However, amygdala activation to fear faces is susceptible to habituation in response during repeated exposure (Breiter et al. 1996) and thus, this could account for reduced amygdala response across the encoding session. This differential habituation in response is extremely unlikely given that only 10 fearful faces were viewed once during the encoding phase. As such, it is unlikely fearful faces are not subject to EEM because of differences in the magnitude of amygdala activation during encoding.

An additional explanation may lie in differences in the precise anatomical localization of amygdala responses to fearful faces and those that support enhanced recollection of other aversive events. The amygdala is composed of many subnuclei with different functions (Gallagher and Holland 1994). Studies from nonhuman animals suggest specifically the basolateral amygdala is critical for the memory-enhancing effects of emotional arousal (McGaugh et al. 1996). Given the less-accurate localization in human neuroimaging, it is unclear whether fearful faces activate the basolateral amygdala or nuclei in a separate amygdala subregion. Consistent with dissociations between fear face recognition and emotional influences on memory (Papps et al. 2003), it is possible that amygdala responses to fearful faces reflect neural activity in nuclei outside of the basolateral nucleus that supports a distinct functional process, which does not result in enhanced memory.

The amygdala responds to specific aspects of fearful facial stimuli, such as the eyes (Whalen et al. 2004; Adolphs et al. 2005a). It may be that enhanced processing of these facial features does not lead to better memory for the overall face. It is important to consider that the amygdala is not the source of the engram supporting episodic memory. A primary role of the amygdala in EEM is to modulate representations in the hippo-

\section{Learning \& Memory \\ www.learnmem.org}


campal formation (Cahill and McGaugh 1998; Hamann et al. 1999; Kilpatrick and Cahill 2003; Dolcos et al. 2004), which are potentially much more complex than what the amygdala specifically encodes. What specific features the amygdala responds to most vigorously are not necessarily equivalent to what is enhanced in later memory. For instance, with regard to mnemonic features, the amygdala has been shown to differentially enhance gist or global representations rather than details (Adolphs et al. 2005b). It is well known that face recognition depends more upon holistic encoding rather than local processing (Farah et al. 1998). Amygdala contributions to face memory would then likely be to enhance global representations that support facial identity recognition. However, precisely how the amygdala interacts with medial temporal-lobe representations of faces and scenes may differ, resulting in the dissociation in EEM shown here.

With regard to psychological factors that may distinguish between memory for faces and scenes is their distinctiveness, which is known to have significant effects on episodic memory (Hunt and Lamb 2001) and may influence the expression of EEM (Dewhurst and Parry 2000). Although viewing expressions of fear may be a highly unique or rare experience, with respect to interitem distinctiveness within the study session, faces are a much more homogenous stimulus class. Scenes were thematically matched with foils to increase the difficulty of the old-new discrimination but likely remained more distinctive than faces, as indicated by the differences in overall memory between the two stimulus types. However, prior studies have shown that amygdala activation is not associated with enhanced memory for distinctive events of little emotional value (Hamann et al. 1999), and behavioral studies have also shown that distinctiveness and emotion appear to have distinct influences on EEM (Anderson et al. 2006). Nonetheless, distinctiveness might be a critical additional factor in conjunction with amygdala activation that allows for the emotional modulation of memory (Strange et al. 2003; Strange and Dolan 2004).

Aversive scenes and fearful faces differed in degree of unpleasantness. Enhanced memory for aversive scenes and not fearful faces may be due to this greater negative valence. Prior studies in rodents have shown the amygdala is involved in aversive as well as appetitive learning (Cahill and McGaugh 1990). The human amygdala responds to events of increased negative as well as positive valence (Hamann et al. 1999, 2002; Anderson et al. 2003b) and similarly, EEM occurs for negative as well as positive events (Hamann et al. 1999). Valence effects on memory are thought to be independent of amygdala function, supported by strategic memory processes supported by the prefrontal cortices (Phelps et al. 1997; Kensinger and Corkin 2004). These effects have greater influence on recall than recognition and are related to the categorical or semantic influences on memory rather than emotional value (Talmi and Moscovitch 2004; Buchanan et al. 2006). As such, the greater negative valence present in aversive scenes relative to fear faces is unlikely to account for the differences in delayed recognition shown here.

We conclude from the present studies that emotional enhancement of memory is not simply reducible to activation of the amygdala. Rather, differential amygdala activation requires other critical conditions to exert neuromodulatory influences on brain regions supporting enhanced recollection. Aversive scenes such as mutilated body parts or horrible car crashes elicit a more intense emotional response than fearful facial expressions, as our self-report (Experiment 1) and peripheral sympathetic measures (Experiment 2) show. A certain threshold of adrenomedullary activation and associated central arousal may be needed in conjunction with amygdala activation to enhance episodic memory. Consistent with the proposed important role of peripheral and central stress hormone release for arousal memory modulation (Kleinsmith and Kaplan 1963; Cahill et al. 1994; Nielson et al. 1996; Cahill and McGaugh 1998; LaBar and Phelps 1998; Buchanan and Lovallo 2001; Hamann 2001; Okuda et al. 2004), fear faces may induce robust amygdala activation, but a level of systemic arousal insufficient for EEM.

Enhanced recollection of emotional events ultimately requires recruitment of the hippocampal formation. While hippocampal activation correlates with memory for nonemotional events, and not the amygdala (Brewer et al. 1998; Wagner et al. 1998; Hamann et al. 1999), conjoint amygdala-hippocampal or parahippocampal activation has been shown for emotionally arousing events (Hamann et al. 1999; Kilpatrick and Cahill 2003; Dolcos et al. 2004). The arousal enhancement of memory traces in the hippocampus is thought to critically depend upon stress steroid activation of the amygdala (McGaugh et al. 1996). In nonhuman animals, heightened emotional arousal during encoding is essential in enabling glucocorticoid effects on memory consolidation (Okuda et al. 2004), with analogous results having been demonstrated in humans (Cahill et al. 2003). Further, blocking of stress steroid $\beta$-adrenergic receptors results in a selective reduction in the emotional enhancement of memory (Cahill et al. 1994; van Stegeren et al. 1998). These findings suggest that enhanced memory associated with emotional experience results from a cascade of neurochemical events linked with peripheral sympathetic activation and the subjective experience of emotionally intense states (Southwick et al. 2002), which in turn enhance hippocampal consolidation of new episodic memories.

Although fearful faces may robustly activate the amygdala, we hypothesize that without stress-steroid receptor binding, these events would not augment the consolidation of hippocampal-based memory traces and facilitate the construction of new episodic memories. We suggest from the present results that enhanced memories for emotionally significant events result from the conjunction of amygdala activation and heightened systemic arousal, allowing the amygdala to influence hippocampaldependent memory traces. Such a mechanism suggests that the greater retention of real-life emotional events would reflect contributions from both the body (systemic arousal) and the brain (amygdala activation).

\section{Materials and Methods}

\section{Experiment 1}

\section{Participants}

Forty-eight students (ages 18-22) either volunteered or were given credit for participation in two studies: face and scene recognition. In each experiment, 16 participants were randomly assigned to one of three delay conditions: 15-min, 1-wk, and 2-wk delay. One subject in the 2 -wk condition was excluded from analysis due to extremely low hit rates. Data files for one participant each in the 1-wk scene condition and 2-wk face condition were corrupted and unavailable for analysis. Informed consent for participation in the study was obtained after the nature of the experimental procedures had been explained.

\section{Stimuli}

Fearful and neutral face stimuli were selected from a variety of publicly available facial affect sets. Eighty photos of 40 individuals (24 male, 16 female) were selected such that there was a photo of each person expressing a fearful and a neutral expression. The 80 photos were then divided into four stimulus sets of 20 unique individuals, with 10 fearful and 10 neutral expressions. These small study sets were necessitated to allow presentation of unique individuals for study and recognition foils. Within each group, there were 12 men and eight women, and half of each gender displayed fearful and half neutral expressions.

Photographic scene stimuli were selected from the Interna- 
tional Affective Picture System (Lang et al. 1988). Twenty photos each of aversive and neutral stimuli were selected and divided into two groups, each containing 10 neutral and 10 negative stimuli. The small study set was used to match number of study items in the face memory task. Negative stimuli were selected on the basis of being unpleasant and highly arousing, based on provided norms. Examples of neutral stimuli included fingerprints, eating utensils, and lamps. Examples of aversive stimuli included dead animals, mutilated bodies, and car crashes.

To characterize the emotional value of the face and scene stimuli, an independent group of 16 participants were asked to judge their valence and arousal responses. These are depicted in Figure 1A in two-factor (valence and arousal) space. Arousal and valence ratings were submitted to two separate $2 \times 2$ ANOVAs, with stimulus type (faces vs. scenes) and emotion (negative vs. neutral) submitted to a separate repeated measures. Consistent with findings of a close association between extremity of negative valence and experiential intensity or arousal (Lang et al 1993), negative scenes were most discrepant on both arousal, $F_{(1,15)}=260.58, P<0.001$, and valence, $F_{(1,15)}=546.51$ $P<0.001$, dimensions relative to all other stimuli. In particular, negative scenes were more arousing, $F_{(1,15)}=119.01, P<0.001$, and negative, $F_{(1,15)}=167.57, P<0.001$, than fearful faces. Fearful faces were more negative, $F_{(1,15)}=54.90, P<0.0001$, and arousing, $F_{(1,15)}=7.60, P<0.02$, than neutral faces.

\section{Encoding task}

Participants sat at the computer where they were first randomly presented with one of the four groups of faces or one of the two groups of scenes. Face presentations were counterbalanced across subjects such that exemplars of fearful expressions in one group displayed neutral expressions in another group, and vice-versa. Half of the participants viewed faces first and the other half viewed scenes first. Encoding of faces and scenes were blocked to avoid overlap in arousal response when intermixed with emotionally aversive scenes. Within each block, all stimuli were presented in a unique random order for each participant. Photos were presented for $3 \mathrm{sec}$ each. Participants were asked to view each stimulus for its entire duration and after its offset to rate their own level of emotional intensity (i.e., arousal). A sevenpoint scale was used where seven was very intense and one not at all intense.

In between face and scene blocks, participants underwent an interpolated distractor task to diffuse potential residual arousal. The distractor task consisted of subjects having to rapidly identify which side of the computer screen was one of two colors selected from various target sets that had to be maintained in memory. The distractor task lasted $\sim 10 \mathrm{~min}$. Stimuli were presented on a computer screen about 2 feet from the subject via PsyScope 1.2.5.

\section{Recognition task}

Participants partook in a recognition memory task $\sim 15 \mathrm{~min}, 1$ wk, or 2 wk following encoding. For face memory, participants were shown sets of 40 faces, 20 of them neutral and 20 of them fearful. Half of the fearful and half of the neutral faces were presented during the initial exposure, and half were new. The new face foils contained pictures of unstudied individuals expressing fear or neutral expressions. For example, if individual A was presented during study with a fear expression, during recognition testing, individual A served as an "old" studied item, and did not appear with a neutral expression as a "new" nonstudied foil. For scene memory, participants were shown sets of 40 pictures: the 20 (10 negative, 10 neutral) pictures from the original exposure, as well as 20 new foils (10 negative, 10 neutral). The two stimulus sets were selected to have thematically similar pictures for each of the neutral (e.g., two household objects) and aversive (e.g., two scenes of car crashes) events in order to have a more stringent and comparable test of recognition memory for neutral and negative events. Participants were presented with the faces and scene blocks in the same order as they were presented during the initial exposure.

After seeing each face or scene, subjects were asked to indi- cate whether the photo (1) was entirely new and had not been presented earlier in the experiment, (2) looked familiar but nothing about it could be specifically retrieved, or (3) remembered specifically that it was presented earlier in the experiment. This differentiation afforded qualitative data about the nature of the retrieved memory (Tulving 1985).

\section{Experiment 2}

\section{Participants}

Twenty-nine undergraduate students (ages 18-22) were recruited to participate in two laboratory sessions, separated by 2 wk. Due to failure to attend a follow-up session and in a few circumstances, equipment errors or aberrant level of autonomic responding, final sample sizes in each analysis were $n=22$ for the galvanic skin-response measurements during encoding and $n=18$ for the delayed recognition memory test. Informed consent for participation in the study was obtained prior to the beginning of the study and the purpose of the experimental procedures was explained during the debriefing at the end of the second experimental session.

\section{Galvanic skin responses (GSR)}

Biopac MP100 series and AcqKnowledge 3.5.2 were used to measure GSR while the subjects were viewing experimental stimuli. Following the method outlined by Cacioppo et al. (2000), two electrodes were placed at the base of the index and middle fingers of a subject's nondominant hand. The sampling frequency for measuring the physiological signals was set at $256 \mathrm{~Hz}$. GSR data were band-pass filtered at $0.1-1 \mathrm{~Hz}$ and smoothed over a $0.5-\mathrm{sec}$ window. First order responses were measured as the maximal increase in the 0.9-4-sec interval following stimulus presentation.

\section{Stimuli and encoding task}

Stimulus selection was similar to Experiment 1. Participants were randomly presented with neutral and fearful faces and scenes. Face presentations were counterbalanced across subjects such that exemplars of fearful expressions in one group displayed neutral expressions in another group, and vice-versa. Events were presented for $4 \mathrm{sec}$ each, and separated by a blank screen that randomly varied in duration from 4 to $6 \mathrm{sec}$. Participants passively viewed each stimulus and returned for a recognition memory test similar to that of Experiment $1 \sim 2 \mathrm{wk}$ after encoding. Stimuli were presented on a computer screen about 2 feet from the subject via E-prime stimulus presentations software.

\section{Acknowledgments}

We thank Rusty Selmont and Peter Wais for assistance with study construction and data collection, John Gabrieli for providing testing resources for Experiment 1, and Jim McGaugh for providing feedback on an earlier version of this manuscript. This study was supported by the National Science and Engineering Research Council (NSERC) of Canada.

\section{References}

Adolphs, R., Tranel, D., Damasio, H., and Damasio, A. 1994. Impaired recognition of emotion in facial expressions following bilateral damage to the human amygdala. Nature 372: 669-672.

Adolphs, R., Cahill, L., Schul, R., and Babinsky, R. 1997. Impaired declarative memory for emotional material following bilateral amygdala damage in humans. Learn. Mem. 4: 291-300.

Adolphs, R., Tranel, D., Hamann, S., Young, A.W., Calder, A.J., Phelps, E.A., Anderson, A., Lee, G.P., and Damasio, A.R. 1999. Recognition of facial emotion in nine individuals with bilateral amygdala damage. Neuropsychologia 37: 1111-1117.

Adolphs, R., Gosselin, F., Buchanan, T.W., Tranel, D., Schyns, P., and Damasio, A.R. 2005a. A mechanism for impaired fear recognition after amygdala damage. Nature 433: $68-72$.

Adolphs, R., Tranel, D., and Buchanan, T.W. 2005b. Amygdala damage impairs emotional memory for gist but not details of complex stimuli. Nat. Neurosci. 8: 512-518.

Akirav, I. and Richter-Levin, G. 2002. Mechanisms of amygdala 
modulation of hippocampal plasticity. J. Neurosci. 22: 9912-9921.

Anderson, A.K. and Phelps, E.A. 2000. Expression without recognition: Contributions of the human amygdala to emotional communication. Psychol. Sci. 11: 106-111.

Anderson, A.K., Spencer, D.D., Fulbright, R.K., and Phelps, E.A. 2000. Contribution of the anteromedial temporal lobes to the evaluation of facial emotion. Neuropsychology 14: 526-536.

Anderson, A.K., Christoff, K., Panitz, D., De Rosa, E., and Gabrieli, J.D. 2003a. Neural correlates of the automatic processing of threat facial signals. J. Neurosci. 23: 5627-5633.

Anderson, A.K., Christoff, K., Stappen, I., Panitz, D., Ghahremani, D.G. Glover, G., Gabrieli, J.D., and Sobel, N. 2003b. Dissociated neural representations of intensity and valence in human olfaction. Nat. Neurosci. 6: 196-202.

Anderson, A.K., Wais, P.E., and Gabrieli, J.D. 2006. Emotion enhances remembrance of neutral events past. Proc. Natl. Acad. Sci. 103: 1599-1604.

Berntsen, D. and Rubin, D.C. 2004. Cultural life scripts structure recall from autobiographical memory. Mem. Cognit. 32: 427-442.

Breiter, H.C., Etcoff, N.L., Whalen, P.J., Kennedy, W.A., Rauch, S.L., Buckner, R.L., Strauss, M.M., Hyman, S.E., and Rosen, B.R. 1996. Response and habituation of the human amygdala during visual processing of facial expression. Neuron 17: 875-887.

Brewer, J.B., Zhao, Z., Desmond, J.E., Glover, G.H., and Gabrieli, J.D. 1998. Making memories: Brain activity that predicts how well visual experience will be remembered. Science 281: 1185-1187.

Broks, P., Young, A.W., Maratos, E.J., Coffey, P.J., Calder, A.J., Isaac, C.L., Mayes, A.R., Hodges, J.R., Montaldi, D., Cezayirli, E., et al. 1998. Face processing impairments after encephalitis: Amygdala damage and recognition of fear. Neuropsychologia 36: 59-70.

Buchanan, T.W. and Lovallo, W.R. 2001. Enhanced memory for emotional material following stress-level cortisol treatment in humans. Psychoneuroendocrinology 26: 307-317.

Buchanan, T.W., al'Absi, M., and Lovallo, W.R. 1999. Cortisol fluctuates with increases and decreases in negative affect. Psychoneuroendocrinology 24: 227-241.

Buchanan, T.W., Etzel, J.A., Adolphs, R., and Tranel, D. 2006. The influence of autonomic arousal and semantic relatedness on memory for emotional words. Int. J. Psychophysiol. 61: 26-33.

Cacioppo, J.T., Tassinary, L.G., and Berntson, G. 2000. Handbook of psychophysiology. Cambridge University Press, New York.

Cahill, L. and McGaugh, J.L. 1990. Amygdaloid complex lesions differentially affect retention of tasks using appetitive and aversive reinforcement. Behav. Neurosci. 104: 532-543.

Cahill, L. and McGaugh, J.L. 1998. Mechanisms of emotional arousal and lasting declarative memory. Trends Neurosci. 21: 294-299.

Cahill, L., Prins, B., Weber, M., and McGaugh, J.L. 1994. $\beta$-adrenergic activation and memory for emotional events. Nature 371: 702-704.

Cahill, L., Babinsky, R., Markowitsch, H.J., and McGaugh, J.L. 1995. The amygdala and emotional memory. Nature 377: 295-296.

Cahill, L., Haier, R.J., Fallon, J., Alkire, M.T., Tang, C., Keator, D., Wu, J., and McGaugh, J.L. 1996. Amygdala activity at encoding correlated with long-term, free recall of emotional information. Proc. Natl. Acad. Sci. 93: 8016-8021.

Cahill, L., Gorski, L., and Le, K. 2003. Enhanced human memory consolidation with post-learning stress: Interaction with the degree of arousal at encoding. Learn. Mem. 10: 270-274.

Canli, T., Zhao, Z., Brewer, J., Gabrieli, J.D., and Cahill, L. 2000. Event-related activation in the human amygdala associates with later memory for individual emotional experience. J. Neurosci. 20: RC99.

Christianson, S.-A., ed. 1992. The handbook of emotion and memory: Research and theory. Lawrence Earlbaum Associates, Hillsdale, NJ.

Dewhurst, S.A. and Parry, L.A. 2000. Emotionality, distinctiveness, and recollective experience. Eur. J. Cogn. Psychol. 12: 541-551.

Dolcos, F., LaBar, K.S., and Cabeza, R. 2004. Interaction between the amygdala and the medial temporal lobe memory system predicts better memory for emotional events. Neuron 42: 855-863.

Farah, M.J., Wilson, K.D., Drain, M., and Tanaka, J.N. 1998. "What is "special" about face perception? Psychol. Rev. 105: 482-498.

Gallagher, M. and Holland, P.C. 1994. The amygdala complex: Multiple roles in associative learning and attention. Proc. Natl. Acad. Sci. 91: $11771-11776$.

Hamann, S. 2001. Cognitive and neural mechanisms of emotional memory. Trends Cogn. Sci. 5: 394-400.

Hamann, S.B., Ely, T.D., Grafton, S.T., and Kilts, C.D. 1999. Amygdala activity related to enhanced memory for pleasant and aversive stimuli. Nat. Neurosci. 2: 289-293.

Hamann, S.B., Ely, T.D., Hoffman, J.M., and Kilts, C.D. 2002. Ecstasy and agony: Activation of the human amygdala in positive and negative emotion. Psychol. Sci. 13: 135-141.

Hariri, A.R., Bookheimer, S.Y., and Mazziotta, J.C. 2000. Modulating emotional responses: Effects of a neocortical network on the limbic system. Neuroreport 11: 43-48.

Hariri, A.R., Tessitore, A., Mattay, V.S., Fera, F., and Weinberger, D.R. 2002. The amygdala response to emotional stimuli: A comparison of faces and scenes. Neuroimage 17: 317-323.

Hunt, R.R. and Lamb, C.A. 2001. What causes the isolation effect? J. Exp. Psychol. Learn. Mem. Cogn. 27: 1359-1366.

Ikegaya, Y., Saito, H., and Abe, K. 1995. Requirement of basolateral amygdala neuron activity for the induction of long-term potentiation in the dentate gyrus in vivo. Brain Res. 671: 351-354.

Kensinger, E.A. and Corkin, S. 2003. Memory enhancement for emotional words: Are emotional words more vividly remembered than neutral words? Mem. Cognit. 31: 1169-1180.

Kensinger, E.A. and Corkin, S. 2004. Two routes to emotional memory: Distinct neural processes for valence and arousal. Proc. Natl. Acad. Sci. 101: 3310-3315.

Kilpatrick, L. and Cahill, L. 2003. Amygdala modulation of parahippocampal and frontal regions during emotionally influenced memory storage. Neuroimage 20: 2091-2099.

Kleinsmith, L.J. and Kaplan, S. 1963. Paired-associate learning as a function of arousal and interpolated interval. J. Exp. Psychol. 65: $190-193$.

LaBar, K.S. and Phelps, E.A. 1998. Arousal-mediated memory consolidation: Role of the medial temporal lobe in humans. Psychol. Sci. 9: 490-493.

Lang, P.J., Öhman, A., and Vaitl, D. 1988. The international affective picture system (slides). Center for Research in Psychophysiology, Univ. of Florida, Gainesville, FL.

Lang, P.J., Greenwald, M.K., Bradley, M.M., and Hamm, A.O. 1993. Looking at pictures: Affective, facial, visceral, and behavioral reactions. Psychophysiology 30: 261-273.

McGaugh, J.L. 2004. The amygdala modulates the consolidation of memories of emotionally arousing experiences. Annu. Rev. Neurosci. 27: 1-28.

McGaugh, J.L., Cahill, L., and Roozendaal, B. 1996. Involvement of the amygdala in memory storage: Interaction with other brain systems. Proc. Natl. Acad. Sci. 93: 13508-13514.

Morris, J.S., Frith, C.D., Perrett, D.I., Rowland, D., Young, A.W., Calder, A.J., and Dolan, R.J. 1996. A differential neural response in the human amygdala to fearful and happy facial expressions. Nature 383: $812-815$

Nielson, K.A., Radtke, R.C., and Jensen, R.A. 1996. Arousal-induced modulation of memory storage processes in humans. Neurobiol. Learn. Mem. 66: 133-142.

Ochsner, K.N. 2000. Are affective events richly recollected or simply familiar? The experience and process of recognizing feelings past. $J$. Exp. Psychol. Gen. 129: 242-261.

Okuda, S., Roozendaal, B., and McGaugh, J.L. 2004. Glucocorticoid effects on object recognition memory require training-associated emotional arousal. Proc. Natl. Acad. Sci. 101: 853-858.

Packard, M.G. and Cahill, L. 2001. Affective modulation of multiple memory systems. Curr. Opin. Neurobiol. 11: 752-756.

Papps, B.P., Calder, A.J., Young, A.W., and O'Carroll, R.E. 2003 Dissociation of affective modulation of recollective and perceptual experience following amygdala damage. J. Neurol. Neurosurg. Psychiatr. 74: 253-254.

Phelps, E.A. and Anderson, A.K. 1997. Emotional memory: What does the amygdala do? Curr. Biol. 7: R311-R314.

Phelps, E.A., LaBar, K.S., and Spencer, D.D. 1997. Memory for emotional words following unilateral temporal lobectomy. Brain Cogn. 35: 85-109.

Phillips, M.L., Young, A.W., Scott, S.K., Calder, A.J., Andrew, C., Giampietro, V., Williams, S.C., Bullmore, E.T., Brammer, M., and Gray, J.A. 1998. Neural responses to facial and vocal expressions of fear and disgust. Proc. Biol. Sci. 265: 1809-1817.

Richter-Levin, G. and Akirav, I. 2000. Amygdala-hippocampus dynamic interaction in relation to memory. Mol. Neurobiol. 22: 11-20.

Sharot, T., Delgado, M.R., and Phelps, E.A. 2004. How emotion enhances the feeling of remembering. Nat. Neurosci. 7: 1376-1380.

Southwick, S.M., Davis, M., Horner, B., Cahill, L., Morgan III, C.A., Gold, P.E., Bremmer, J.D., and Charney, D.C. 2002. Relationship of enhanced norepinephrine activity during memory consolidation to enhanced long-term memory in humans. Am. J. Psychiatry 159: $1420-1422$

Strange, B.A. and Dolan, R.J. 2004. $\beta$-adrenergic modulation of emotional memory-evoked human amygdala and hippocampal responses. Proc. Natl. Acad. Sci. 101: 11454-11458.

Strange, B.A., Hurlemann, R., and Dolan, R.J. 2003. An emotion-induced retrograde amnesia in humans is amygdala- and $\beta$-adrenergic-dependent. Proc. Natl. Acad. Sci. 100: 13626-13631.

Talmi, D. and Moscovitch, M. 2004. Can semantic relatedness explain the enhancement of memory for emotional words? Mem. Cognit. 32: $742-751$. 


\section{Anderson et al.}

Tulving, E. 1985. Memory and consciousness. Can. Psychol. 26: 1-12.

van Stegeren, A.H., Everaerd, W., Cahill, L., McGaugh, J.L., and Gooren, L.J. 1998. Memory for emotional events: Differential effects of centrally versus peripherally acting $\beta$-blocking agents. Psychopharmacology 138: 305-310.

Wagner, A.D., Schacter, D.L., Rotte, M., Koutstaal, W., Maril, A., Dale, A.M., Rosen, B.R., and Buckner, R.L. 1998. Building memories: Remembering and forgetting of verbal experiences as predicted by brain activity. Science 281: 1188-1191.

Whalen, P.J., Rauch, S.L., Etcoff, N.L., McInerney, S.C., Lee, M.B., and Jenike, M.A. 1998. Masked presentations of emotional facial expressions modulate amygdala activity without explicit knowledge. J. Neurosci. 18: 411-418.

Whalen, P.J., Shin, L.M., McInerney, S.C., Fischer, H., Wright, C.I., and
Rauch, S.L. 2001. A functional MRI study of human amygdala responses to facial expressions of fear versus anger. Emotion 1: 70-83.

Whalen, P.J., Kagan, J., Cook, R.G., Davis, F.C., Kim, H., Polis, S.,

McLaren, D.G., Somerville, L.H., McLean, A.A., Maxwell, J.S., et al. 2004. Human amygdala responsivity to masked fearful eye whites. Science 306: 2061.

Yonelinas, A.P. and Levy, B.J. 2002. Dissociating familiarity from recollection in human recognition memory: Different rates of forgetting over short retention intervals. Psychon. Bull. Rev.

9: $575-582$.

Received August 16, 2005; accepted in revised form August 23, 2006. 


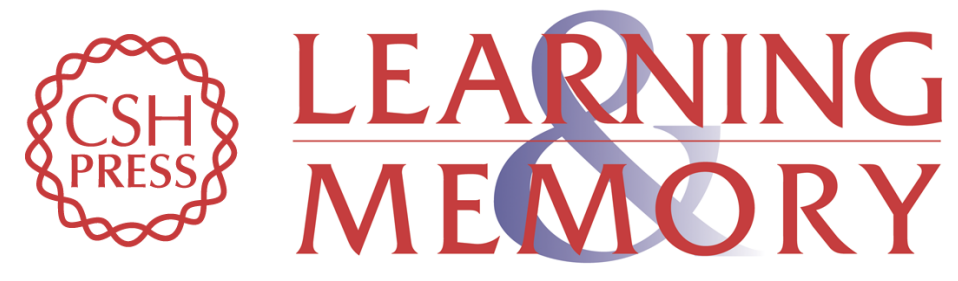

\section{Emotional memories are not all created equal: Evidence for selective memory enhancement}

Adam K. Anderson, Yuki Yamaguchi, Wojtek Grabski, et al.

Learn. Mem. 2006, 13: originally published online November 13, 2006

Access the most recent version at doi:10.1101/lm.388906

References This article cites 65 articles, 17 of which can be accessed free at:

http://learnmem.cshlp.org/content/13/6/711.full.html\#ref-list-1

License

Email Alerting Receive free email alerts when new articles cite this article - sign up in the box at the Service top right corner of the article or click here. 